\title{
A Multi-Objective Optimization Method for Hospital Admission Problem-A Case Study on Covid-19 Patients
}

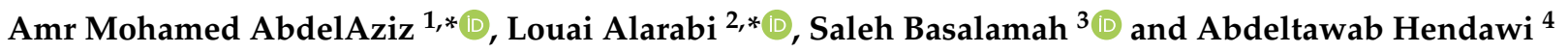 \\ 1 Faculty of Computers and Artificial Intelligence, Beni-Suef University, Bani Sweif 62511, Egypt \\ 2 Department of Computer Science, Umm Al-Qura University, Mecca 21421, Saudi Arabia \\ 3 Department of Computer Engineering, Umm Al-Qura University, Mecca 21421, Saudi Arabia; \\ smbasalamah@uqu.edu.sa \\ 4 Department of Computer Science and Statistics, University of Rhode Island, Kingston, RI 02881, USA; \\ hendawi@uri.edu \\ * Correspondence: amraziz@fcis.bsu.edu.eg (A.M.A.); lmarabi@uqu.edu.sa (L.A.)
}

check for updates

Citation: AbdelAziz, A.M.; Alarabi, L.; Basalamah, S.; Hendawi, A. A Multi-Objective Optimization Method for Hospital Admission Problem-A Case Study on Covid-19 Patients. Algorithms 2021, 14, 38. https://doi.org/10.3390/a14020038

Academic Editor:Massimiliano

Caramia

Received: 19 December 2020

Accepted: 25 January 2021

Published: 27 January 2021

Publisher's Note: MDPI stays neutral with regard to jurisdictional clai$\mathrm{ms}$ in published maps and institutional affiliations.

Copyright: $(\odot 2021$ by the authors. Licensee MDPI, Basel, Switzerland. This article is an open access article distributed under the terms and conditions of the Creative Commons Attribution (CC BY) license (https:// creativecommons.org/licenses/by/ $4.0 /)$.

\begin{abstract}
The wide spread of Covid-19 has led to infecting a huge number of patients, simultaneously. This resulted in a massive number of requests for medical care, at the same time. During the first wave of Covid-19, many people were not able to get admitted to appropriate hospitals because of the immense number of patients. Admitting patients to suitable hospitals can decrease the in-bed time of patients, which can lead to saving many lives. Also, optimizing the admission process can minimize the waiting time for medical care, which can save the lives of severe cases. The admission process needs to consider two main criteria: the admission time and the readiness of the hospital that will accept the patients. These two objectives convert the admission problem into a Multi-Objective Problem (MOP). Pareto Optimization (PO) is a common multi-objective optimization method that has been applied to different MOPs and showed its ability to solve them. In this paper, a PO-based algorithm is proposed to deal with admitting Covid-19 patients to hospitals. The method uses PO to vary among hospitals to choose the most suitable hospital for the patient with the least admission time. The method also considers patients with severe cases by admitting them to hospitals with the least admission time regardless of their readiness. The method has been tested over a real-life dataset that consisted of 254 patients obtained from King Faisal specialist hospital in Saudi Arabia. The method was compared with the lexicographic multi-objective optimization method regarding admission time and accuracy. The proposed method showed its superiority over the lexicographic method regarding the two criteria, which makes it a good candidate for real-life admission systems.
\end{abstract}

Keywords: multi-objective optimization; smart health; hospital use

\section{Introduction}

The unprecedented spread of the Severe Acute Respiratory Syndrome Coronavirus 2 (Covid-19) epidemic across the world has pushed the healthcare systems to their limits [1,2]. The main problem comes from having too many patients that need medical assistance at the same time [3]. Infected patients vary in their status regarding their stage in the infection and their previous illnesses, medically known as (comorbidities). Having an effective admission system for Covid-19 patients can alleviate the burden over hospitals through admitting patients to the most suitable hospital according to their status in real time. This can minimize the waiting time of patients and save patients with severe status. To our knowledge, few studies have been proposed to deal with this problem [4].

Identifying the most suitable hospital for a patient to be admitted automatically is not an easy task. This task has parameters that need to be considered, such as an assessment of patient status, assessment of hospital preparations, the time before admitting patients to hospitals, and time that patients need to reach the hospital. This transforms the admission problem into a Multi-Objective Problem (MOP). Solving MOPs needs multiple solutions 
that vary the degree of satisfaction to different objectives [5]. Identifying the best solution to the problem can be determined according to the current status of the problem.

Pareto Optimization $(P O)$ has been one of the most common methods used to solve $M O P s$ [6]. It tries to satisfy the whole objectives simultaneously. It generates a nondominated set of solutions for MOPs, which cannot be further optimized. $P O$ has been applied to solve MOPs in different fields, such as clustering [7-9] and hospital resource optimization [10-12] $P O$ can be employed to solve the admission problem considering the different objectives of the problem as it has been used before in [13].

In this paper, a multi-objective algorithm is proposed to provide automatic identification of the appropriate hospital for Covid-19 patients according to their medical condition in real time. The main contributions of this paper can be summarized in:

- Develop an effective multi-objective admission system for Covid-19 patients that admits patients to the most suitable hospitals in real time and considers the comorbidities of the patients.

- The method considers two main criteria in the admission process: (1) the patient status regarding the hospital preparations and (2) the admission time (reach time and admission time). This method can minimize the in-bed time of patients as it directs each patient to the most suitable hospital.

- Provide a mathematical representation of the problem and the main constraints that affect it.

- Implement the method using the $P O$ to vary among the conflicting objectives as admitting a patient to a non-suitable hospital in less time can result in transferring the patient to a different one.

- Test the method over a dataset that combines a real-life part that has been provided by King Faisal specialist hospital in Saudi Arabia and a synthetic part. The real-life part had the clinical symptoms of the patients and their medical conditions when they arrived at the hospital. Meanwhile, the arrival rate, admission time, time to reach, and medical devices in different hospitals have been generated randomly to mimic the real-life situation.

- Results show the efficiency of $P O$ in obtaining the correct hospital for patients over the Lexicographic method [7]. Also, the method showed its effectiveness in obtaining the correct hospital in real time despite the increase in the number of hospitals.

The rest of the paper is organized as follows. Section 2 presents the recently published papers that focused on the problem under investigation. Section 3 presents a detailed description of MOPs, $P O$, and mathematical definitions of the admission problem and its constraints. Section 4 describes the proposed method, gives the pseudocode of the algorithm, presents a tracing example of the execution of the method over a specific patient, and gives a complexity analysis of the proposed algorithm. Section 5 shows a description of the dataset and the different experiments that have been conducted to prove the effectiveness of the proposed method. Finally, Section 6 presents a conclusion of the proposed method and future directions related to this problem.

\section{Related Work}

Using hospital resources is not a new problem, multiple researchers have proposed different solutions for this problem from different perspectives. Some researchers focused on optimizing hospital operation rooms to maximize the number of operations performed each year. Abedini et al. proposed a mechanism to produce a schedule that can ensure the number of patients in each stage does not exceed the number of beds in that stage, which minimized patients' blocking [14]. Another perspective has been proposed for the optimization of the healthcare system, which focused on the internships of doctors in hospitals [15]. The authors proposed a method to control the admission of doctors of internships in hospitals according to two criteria: the needs of the regions that the hospitals existed into and the preferences of doctors. They applied a Multi-Objective Optimization (MOO) method to find the best solutions for the MOP. 
Multiple studies have been proposed to control the patients' admission to hospitals while considering the maximization of the number of patients to be served and the minimization of the waiting time of patients $[10,16-18]$. These studies focused on maximizing the use of beds in each hospital by generating a schedule that can guarantee the availability of beds and minimizing the hospitalization time of patients. The authors employed a $M O O$ method to obtain the best schedule that can satisfy the different objectives assumed during the implementation. Due to the uncertainty of patients' arrival to hospitals, those methods resulted in having multiple queues of waiting patients. Belciug et al. [19] proposed an improved use method to minimize the number of patients in the waiting queues. The authors applied an evolutionary-based model to manage the patients' admission and bed allocation to minimize the number of patients in waiting queues.

From the perspective of admitting patients to hospitals, different studies have been proposed [4,20-22]. Anselmi et al. illustrated the importance of the fast response of an emergency system through the arrival time of ambulances on the number of deaths of patients [20]. Because of the importance of the admission problem, Pope et al. conducted research to identify the non-clinical factors that can affect the admission of patients [22]. Considering the previous method, it is clear that converting the admission problem into a $M O P$ is new and has not been proposed before.

In the next section, a detailed description of MOPs and $P O$ are given. Also, a mathematical representation of the admission problem and the proposed methodology to solve the problem are given.

\section{Background}

3.1. Multi-Objective Problems (MOPs)

A $M O P$ can be described as a set of $m$ objectives as:

$$
F=\min \left[f_{1}, f_{2}, \ldots, f_{m}\right],
$$

where $m$ represents the number of objective functions of the MOP. Each objective can be described mathematically as a set of equality or inequality equations as [23]:

$$
\begin{array}{ll}
H_{i}(x)=0 ; & i=1, \ldots, k, \\
G_{j}(x) \leq 0 ; & j=1, \ldots, t
\end{array}
$$

where $H$ represents the equality functions and the $G$ represents the inequality functions. These functions assess the quality of solutions. Each parameter of an objective has a search space that can be described as:

$$
X_{i}^{l} \leq X_{i} \leq X_{i}^{u} ; \quad i=1, \ldots, n,
$$

where $n$ is the number of variables, $l$ and $u$ represent the lower and upper bounds of each parameter $i$ respectively. There are a limited number of algorithms that can deal with $M O P s$, which include $P O$ [6] and the lexicographic method [7]. The lexicographic approach starts with ordering the objectives of the problem according to their importance. Then, it tries to find the optimal solution for the problem according to the order of the objectives.

\subsection{Pareto Optimization}

$P O$ is a common method that has been widely used to solve MOPs $[6,8,12,23]$. It tries to satisfy the whole objectives simultaneously. $P O$ can compare among multiple solutions of a $M O P$ based on different objectives and identify the non-dominated solutions or the Pareto Front $(P F)$ solutions. The PF or non-dominated solutions are the solutions that cannot be further optimized. Optimizing one objective in the non-dominated solutions results in deteriorating the degree of optimization of other objectives.

$P O$ applies the dominance concept between two solutions $v$ and $z$ by varying among the objectives in two solutions, in case all objectives in $v$ are as good as in $z$ and at least 
one objective in $v$ is better than the same objective in $z$, then solution $v$ is considered to dominate solution $z$. This comparison can be described mathematically as [5]:

$$
\begin{aligned}
& f_{i}(v) \leq f_{i}(z) ; \quad \forall i \quad \epsilon[1, m] \\
& f_{i}(v)<f_{i}(z) ; \quad \exists i \quad \epsilon[1, m]
\end{aligned}
$$

where $v, z$ are two different solutions, and $m$ represents the number of objectives. These two rules must hold to ensure that solution $v$ dominates solution $z$.

\subsection{Problem Definition}

The admission problem of Covid-19 patients to hospitals can be formulated of two objectives: the first objective is concerned with the time that a patient needs to get admitted and the second objective is concerned with matching the patient status with the hospital medical preparations. The first objective has two main factors: the time that a patient needs to reach a hospital $t_{r}$ and the admission time of the patient to get the medical care $t_{a d}$. The second objective focuses on finding the hospital, equipped with the best medical devices $h_{m d}$ compared with the patient comorbidities $p_{\text {com }}$. De Nardo et al. [24] have identified 11 comorbidities to prioritize the patients infected with Covid-19 virus. The proposed model has used seven comorbidities of them, which include diabetes mellitus, heart failure, chronic pulmonary disease, chronic liver disease, chronic kidney disease, Temperature, and $\mathrm{O}_{2}$ saturation. These comorbidities are used to evaluate the patient's status to identify the devices that should be in a hospital to treat the patients.

The best solution to the problem can be identified as the hospital $h$ with the least admission $h_{t_{a d}}$, reach $h_{t_{r}}$ times, and has the most suitable medical devices $h_{t_{m d}}$ for the patient's comorbidities $P_{\text {com }}$. The problem has a set of constraints, which can be summarized into the existence of a limited number of hospitals $h=\{1,2, \ldots, H\}$, a limited number of medical devices in each hospital $m d=\{1,2, \ldots, M D\}$, and the response time of the system to admit patients should not exceed a specific threshold.

To choose the best hospital for patient admission, the hospital should have the most suitable medical devices for the patient and does not require a long time to admit the patient. This clarifies the existence of two conflicting objectives that a solution needs to satisfy. The first objective is related to the admission time, which is a minimization function. The second objective is concerned with assessing how suitable the medical devices in a hospital compared with the patient status, which is a maximization function. According to this scenario, the problem can be defined as:

$$
\left\{\begin{array}{l}
T_{p}(h)=\operatorname{Maximum}\left(h_{t_{a d}}, h_{t_{r}}\right) \\
F_{p}(h)=\operatorname{Match}\left(h_{m d}, P_{c o m}\right) \quad \text { Maximimization }
\end{array}\right.
$$

The first function is used to identify the nearest hospital to the patient location and has the least admission time based on two times: reach time $h_{t_{a d}}$ and admission time $h_{t_{r}}$. This function takes the maximum time of the two times $\left(h_{t_{r}}, h_{t_{a d}}\right)$ to determine the time needed for a patient to be admitted to hospital $h$. The second function is applied to identify the hospital that has the maximum number of medical devices needed to treat the patient. This ensures that the patient will have the whole medical care needed in one hospital. The matching function makes a comparison of the patient's comorbidities with the medical devices of each hospital. PO can be applied to obtain the whole non-dominated solutions to the problem. The best solution will be determined from the non-dominated solutions based on each patient's situation. To describe the main parameters used in the problem, Table 1 presents the notations of these parameters and their description. 
Table 1. A description of the main parameters of the problem definition.

\begin{tabular}{cc}
\hline Parameter & Description \\
\hline$h_{t_{a d}}$ & the time needed for a patient to be admitted in a hospital \\
$h_{t_{r}}$ & the time needed for a patient to reach a hospital location \\
$h_{m d}$ & the medical devices possessed by a hospital \\
$P_{c o m}$ & the comorbidities of a patient \\
$t$ & the time at which the event happens \\
$d_{p t}$ & the demand of patient $p$ in time $t$ \\
$h_{m b}$ & the maximum number of beds in a hospital $h$ \\
$M H B$ & maximum number of beds in hospitals \\
$R_{h p t}$ & the response time of the system to identify a hospital $h$ for patient $p$ \\
\hline
\end{tabular}

\subsection{Problem's Constraints}

From our perspective, the main constraints of the admission problem can be concluded into: (1) the number of available beds in a hospital at time $t$ and (2) the admission time should be minimum. This analysis has been deducted based on multiple recent studies $[17,25,26]$. As we can see in each method, different constraints are considered. This returns to the problem itself and the perspective of the authors to the problem. The first constraint can be mathematically defined as:

$$
\sum_{p \in P} p_{c o m} \leq h_{m b} \quad \forall t \in T
$$

This function ensures that the total number of patients' requests do not exceed the total number of beds in all the hospitals $M H B$. In case the number of requests exceeds the total number of beds, the patients' requests are added to a queue to be handled later.

Another important constraint is the response time of the system to identify the most suitable hospital for the patient. This can be defined mathematically as:

$$
R_{h p t}-d_{p t} \leq \alpha \quad \forall p \in P,
$$

where $p$ represents the patient, $h$ is the identified hospital, and $d_{p t}$ is the demand of patient $p$ at time $t$. This constraint ensures that patients should not wait for more than the threshold $\alpha$ to know to which hospital they would be admitted.

\section{Methodology}

\subsection{The Objective Function}

In the proposed method, the objective function is based on identifying the most suitable hospital regarding the patient's status and the admission time. The admission time of a hospital $H$ is computed as the maximum time $M a x_{t}$ of reach time $H 1_{t_{r}}$ and admission time for the patient to get medical care $H 1_{t_{a d}}$. The patient status is represented by $P_{c o m}$, while the preparation of hospital $H 1$ is denoted by $H 1_{m d}$. A matching criterion has been developed to identify each symptom with patient status. The match function generates a number that shows the degree of readiness that the hospital must receive the patient. For each hospital, a solution is constructed of hospital number, match value, and admission time. Based on the three criteria, the method varies among different hospitals.

The method considers patients with severe conditions as it directs those patients to hospitals with the least admission time regardless of the readiness of the hospital to the patient status. This can save the patient's life because he/she needs immediate medical attention. For patients with non-severe cases, they are directed to hospitals with maximum match value and smallest admission time. The method considers the appropriateness of the hospital as the first objective to be optimized, then the admission time. The way to 
compute objective function for two hospitals $\mathrm{H} 1, \mathrm{H} 2$, and the identification of the best hospital is given as:

$$
\begin{array}{r}
H 1_{\text {Max }_{t}}=\operatorname{Max}\left(H 1_{t_{r}}, H 1_{t_{a d}}\right) \\
H 1_{m}=\operatorname{Match}\left(P_{\text {com }}, H 1_{m d}\right) \\
H 1=\left(H 1_{m}, H 1_{\text {Max }_{t}}\right) \\
H 2=\left(H 2_{m}, H 2_{\text {Max }_{t}}\right)
\end{array}
$$

The $H 1$ hospital can be identified to dominate $H 2$ if the readiness value of $H 1$ is bigger than or equal to the value of $H 2$ and the admission time of $H 1$ is less than or equal to the admission time of $H 2$. This can be represented mathematically as:

$$
H 1_{m} \geq H 2_{m} \text { and } H 1_{\text {Maxt }_{t}} \leq H 2_{\text {Max }_{t}}
$$

\subsection{The Proposed Algorithm}

$P O$ has been applied to solve different $M O P$ s. It can be used to determine the best hospital for a patient to be admitted according to (6). $P O$ can obtain the best set of hospitals for patient admission based on the dominance criterion. The ability of $P O$ to vary among different solutions based on multiple criteria allows the algorithm to obtain the best hospital that has the highest matching score and the least admission time.

The proposed Algorithm 1 starts with an admission request of a patient $p$ with comorbidities $P_{\text {com }}$. The method traverses all the hospitals to evaluate the time to admit the patient and to assess the preparations of each hospital. The method considers the admission time to be the maximum time of admission time $t_{a d}$ and time to reach the hospital $t_{r}$. The matching function compares the patient's comorbidities and medical devices in the hospital to assess the readiness of the hospital to admit the patient. Considering the two criteria, the algorithm uses the $P O$ dominance to decide whether to add this solution to the nondominated solutions or not. The algorithm ends with a set of best solutions stored in the non-dominated set. The method considers the severity of the patient so, it chooses the hospital with the least admission time regardless of the medical devices of the hospital. This ensures that the patient can have urgent medical care to prevent his status from sudden death. If the patient's status is not severe, he/she will be admitted to the most suitable hospital for his status and with the least admission time. In the waiting time, the patient will be advised to stay in quarantine until admission time. A graphical representation of the steps of the proposed algorithm is shown in Figure 1.

\subsection{A Tracing Example of the Algorithm}

In the following, a tracing example is given for a certain patient that has comorbidities according to Table 2. The patient is diabetic with a mild increase in body temperature (37.8) and his $\mathrm{O}_{2}$ saturation is 96 . The patient is not known to have any other diseases.

This example has been tested over nine hospitals that have medical preparations regarding kidney machines, ventilators, and Intensive Care Units (ICUs) according to Table 3. Also, Table 3 presents the time to reach and time to admit to the patient under the test. In our experiments, the time to reach and time to admit are stored in the patient record as these times differ for each patient. Table 3 has the number of available beds that can accept the patients, which is used to determine whether the hospital is full or not.

Table 4 presents a tracing example of the execution of the algorithm over the patient identified in Table 2 to the hospitals presented in Table 3. Table 4 gives in each row an execution step of the algorithm. It shows the iteration number, reach time of the patient to the hospital, admission time of the patient to the hospital, maximum of reach and admission times, match value of patient comorbidities and hospital preparations, and the best hospitals to admit the patient. 


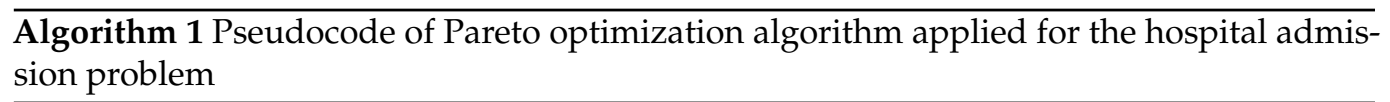

\section{Initialization.}

Let the number of patients $P_{n o}$, number of hospitals $H_{n o}$, patient's comorbidities $P_{\text {com }}$, BestSol to be the best solution, $h_{r}$ time for the patient to reach the hospital, $t_{a d}$ the time needed for the patient to be admitted in the hospital, and empty the non-dominated set of solutions.

\section{Main Loop.}

$$
\begin{aligned}
& \text { for } p=1, \ldots, P_{n o} \text { do } \\
& \text { for } h=1, \ldots, H_{n o} \text { do } \\
& \quad \text { Set } \operatorname{Max} x_{t}=\operatorname{Max}\left(h_{t_{r}}, h_{t_{a d}}\right) \\
& \text { Set } m=\operatorname{Match}\left(P_{c o m}, H(h)_{m d}\right)
\end{aligned}
$$

Set Sol to be the set of $\left(h, m, M a x_{t}\right)$.

Add Sol to non-dominated solutions according to dominance criteria of $P O$.

\section{end for}

\section{if $P\left(p_{\text {status }}\right) \quad$ is Severe then}

Set BestSol to be the hospital with the smallest admission time Max $x_{t}$ regardless of the patient's comorbidities and the hospital's medical devices.

else time.

Set BestSol to be the hospital with the highest matching score and least admission

$$
\text { end if }
$$

end for

Return BestSol

In iteration 1, the maximum time of reach and admission is 10 and the match value is 1 between patient and hospital. The hospital number is considered to be the best hospital and is added to the non-dominated solutions because there are no other solutions. At iteration 2, hospital number 2 has the same maximum time and match value so, the nondominated list adds hospital number 2 . At iteration number 3, hospital 3 has a maximum time of 8 and zero matching criterion. As hospital 3 has less time, it will be added to the non-dominated set without considering the zero-match value as this hospital could be valid for severe cases. The rules of accepting the previous hospital or not can be changed according to the real-life decision-makers. Iteration 4 has the same case as in iterations $(1,2)$. At iteration number 5, the hospital is rejected as it has a maximum time greater than those in the non-dominated solutions. Hospitals number $(6,7)$ are rejected as their maximum time is greater than the maximum time of hospital number 3. Hospital number 8 has been added to the non-dominated solutions and eliminated hospital number 3 as it has zero matches and has less maximum time than hospital number 3 . At iteration number 9 , the hospital dominates the hospitals $(1,2,4)$ as it has the same match value and has less maximum time. 


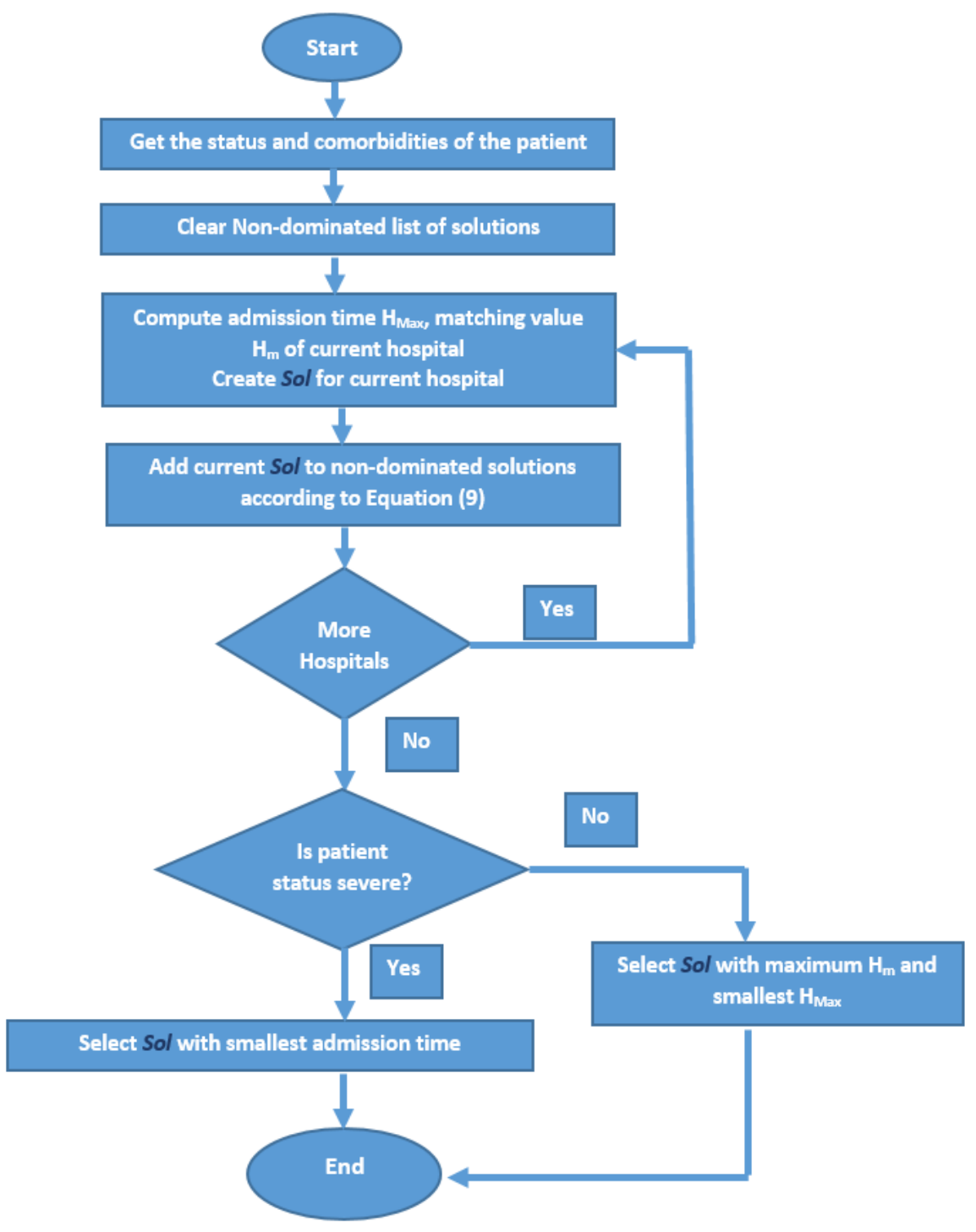

Figure 1. A depiction of the proposed method.

Table 2. Patient's comorbidities.

\begin{tabular}{ccccccc}
\hline Diabetes Mellitus & Heart Failure & $\begin{array}{c}\text { Chronic Pulmonary } \\
\text { Disease }\end{array}$ & $\begin{array}{c}\text { Chronic Liver } \\
\text { Disease }\end{array}$ & $\begin{array}{c}\text { Chronic Kidney } \\
\text { Disease }\end{array}$ & $\begin{array}{c}\text { Temp } \\
\text { Saturation }\end{array}$ \\
\hline 1 & 0 & 0 & 0 & 0 & 37.8 & 96 \\
\hline
\end{tabular}

Table 3. The medical preparations, reach times, and admission times of nine hospitals.

\begin{tabular}{|c|c|c|c|c|c|c|}
\hline Num. & Kidney Machine & Ventilator & Intensive Care Unit & Time to Reach & Time to Admit & Available Beds \\
\hline 1 & 1 & 1 & 1 & 3 & 10 & 39 \\
\hline 2 & 1 & 1 & 1 & 2 & 10 & 10 \\
\hline 3 & 1 & 0 & 1 & 3 & 8 & 34 \\
\hline 4 & 0 & 1 & 1 & 5 & 10 & 31 \\
\hline 5 & 1 & 1 & 0 & 5 & 15 & 9 \\
\hline 6 & 1 & 1 & 0 & 6 & 12 & 55 \\
\hline 7 & 0 & 0 & 1 & 4 & 9 & 3 \\
\hline 8 & 1 & 0 & 1 & 1 & 6 & 43 \\
\hline 9 & 0 & 1 & 0 & 1 & 9 & 16 \\
\hline
\end{tabular}


Table 4. A tracing example of admitting the patient considering the nine hospitals.

\begin{tabular}{cccccc}
\hline Iter. Num & Reach Time & Admission Time & Maximum Time & Match Value & Best Solutions \\
\hline 1 & 3 & 10 & 10 & 1 & $(1)$ \\
2 & 2 & 10 & 10 & 1 & $(1,2)$ \\
3 & 3 & 8 & 8 & 0 & $(1,2,3)$ \\
4 & 5 & 10 & 10 & 1 & $(1,2,3,4)$ \\
5 & 5 & 15 & 15 & 1 & $(1,2,3,4)$ \\
7 & 6 & 12 & 9 & 0 & $(1,2,3,4)$ \\
8 & 4 & 9 & 6 & 0 & $(1,2,3,4)$ \\
9 & 1 & 6 & 9 & 1 & $(1,2,4,8)$ \\
\hline
\end{tabular}

The previous Table 4 presented the tracing example of 9 hospitals, which has finished with identifying the best hospitals 8,9 . If the patient's status is not severe, he/she will be admitted to hospital number 9 , otherwise, he/she will be admitted to hospital number 8 . Figure 2 depicts the tracing example by presenting the patient and the objective values of each hospital.

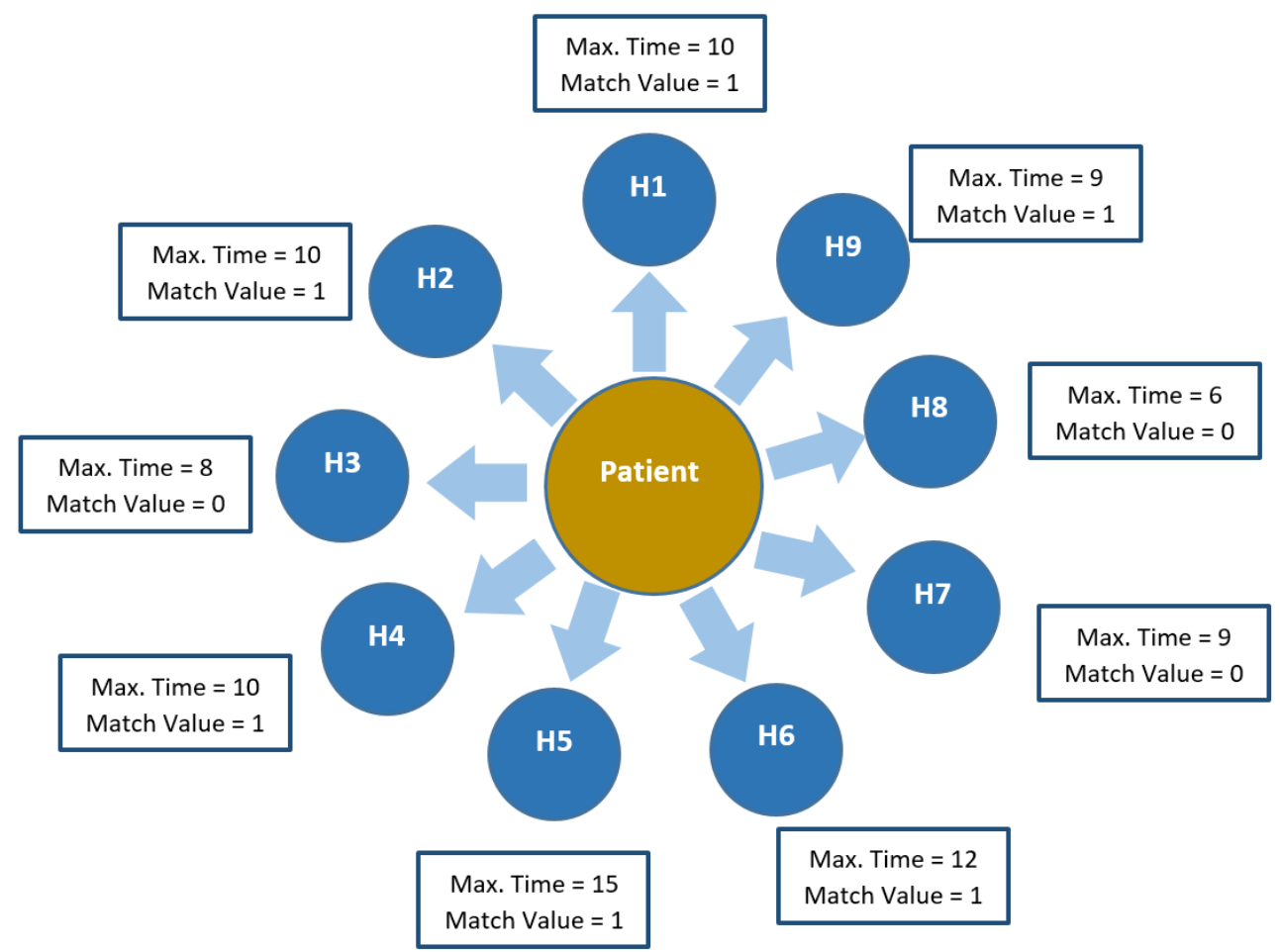

Figure 2. A graphical representation of the objective values of the hospitals after computing them relative to the patient's status.

\subsection{A Complexity Analysis of the Method}

From the first look, the complexity analysis of the proposed Algorithm 1 can be evaluated as $P \times H$, where $P$ represents the number of patients and $H$ represents the number of hospitals. In real-life, the $P$ will represent only the patient that asks for the admission request and $H$ will still represent the number of the whole hospitals. Therefore, the complexity analysis can be determined as the number of hospitals only.

In the next section, detailed numerical experiments will be given to assess the efficiency of the method over a real-life dataset. 


\section{Experimental Results}

Several experiments have been conducted over a real-life dataset to evaluate the effectiveness of the proposed method in admitting Covid-19 patients to suitable hospitals. The algorithm was programmed in Scala in a Linux environment. The real-life dataset had been provided by the King Faisal specialist hospital for Covid-19 patients [27]. The dataset contained information about demography, exposure, vaccination history, and comorbidities of the admitted patients. A set of comorbidities has been chosen from the original dataset according to [24] to assess the medical status of patients. These comorbidities have been used to identify the medical devices needed for each set of comorbidities.

The admission times of hospitals and reach times of patients to hospitals have been generated randomly according to the Poisson distribution, which has been used before to mimic the random calls for emergency services in [28]. The medical devices in each hospital have been generated randomly according to normal distribution. The kidney machine has been identified to be necessary for patients that have chronic kidney disease or any serious kidney illnesses. The $\mathrm{O}_{2}$ Oxygen inhalation/Ventilator has been identified to be mandatory for patients that have $\mathrm{O}_{2}$ saturation less than 92 or have chronic pulmonary diseases. The ICU has been identified to be mandatory for patients that have chronic liver disease, comma, and Heart Failure. These criteria can be extended or changed concerning the new studies about Covid-19.

The dataset was constructed of 254 patients, each had 7 comorbidities. Table 5 presents different times (minimum time, average time, maximum time) that the algorithm needs to admit each patient to the most suitable hospital, where the time is evaluated in seconds. Results in Table 5 present that the algorithm can admit patients over the different number of hospitals in real time. Even the maximum time is suitable for real-life severe cases as it does not reach a single second.

Table 5. Different times (seconds) needed to admit each patient to a hospital over a different number of hospitals.

\begin{tabular}{cccc}
\hline & Minimum Time & Average Time & Maximum Time \\
\hline Preto Optimization & & & \\
\hline 20 Hospitals & 0 & $3.68 \times 10^{-6}$ & 0.000215 \\
30 Hospitals & 0 & $6.59 \times 10^{-6}$ & $2.05 \times 10^{-4}$ \\
40 Hospitals & 0 & $7.53 \times 10^{-6}$ & $2.46 \times 10^{-4}$ \\
50 Hospitals & 0 & $7.71 \times 10^{-6}$ & $2.26 \times 10^{-4}$ \\
60 Hospitals & 0 & $1.23 \times 10^{-5}$ & $2.48 \times 10^{-4}$ \\
\hline lexicographic Method & & & \\
\hline 20 Hospitals & 0 & $4.05 \times 10^{-6}$ & 0.000226 \\
30 Hospitals & 0 & $7.37 \times 10^{-6}$ & $2.01 \times 10^{-4}$ \\
40 Hospitals & 0 & $2.00 \times 10^{-5}$ & $4.25 \times 10^{-4}$ \\
50 Hospitals & 0 & $1.94 \times 10^{-5}$ & $3.64 \times 10^{-4}$ \\
60 Hospitals & 0 & $2.98 \times 10^{-5}$ & 0.0016217 \\
\hline
\end{tabular}

Figure 3 presents a graphical comparison between the proposed algorithm using $P O$ and the lexicographic method to vary among a different number of hospitals. The graph shows the average running time of the two methods, evaluated into seconds. The figure shows that the average running time of the lexicographic increases with the increase in the number of hospitals. However, the increase in the proposed method is very small even with the increase in the number of hospitals. This emphasizes the stability of the proposed method as it keeps a near steady performance despite the number of hospitals. 


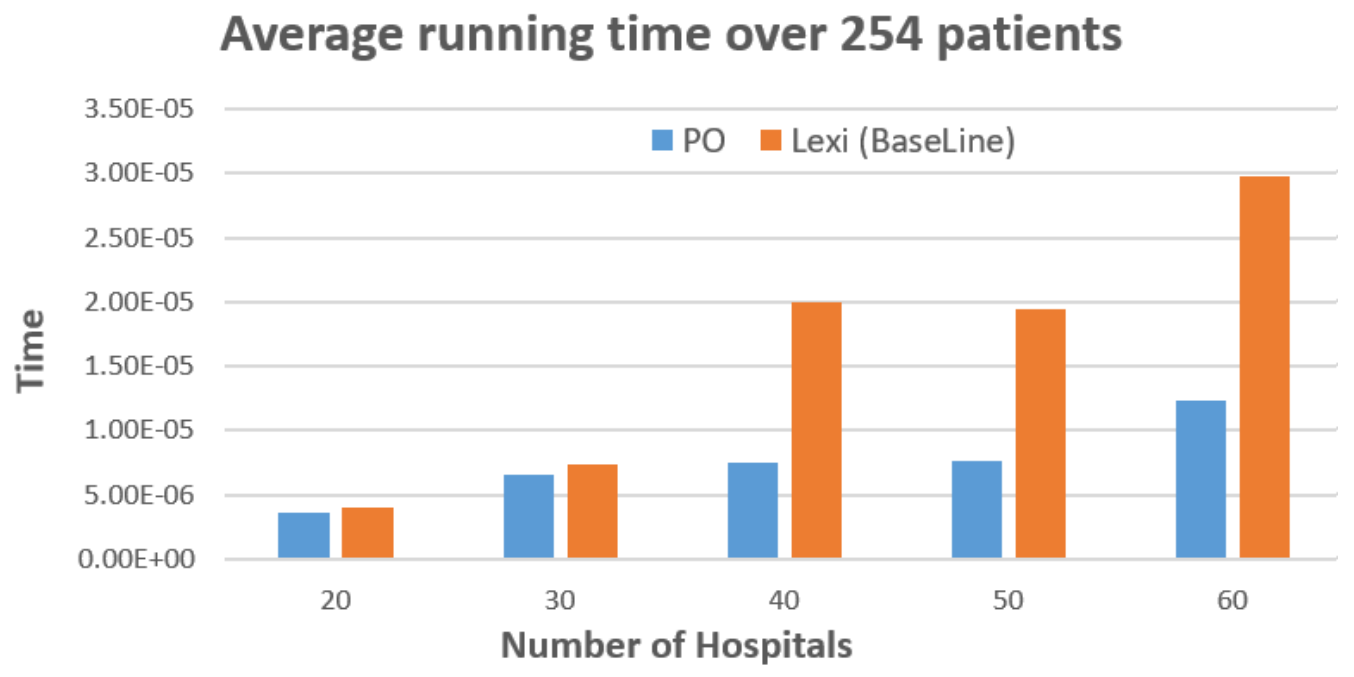

Figure 3. A comparison between Pareto optimization and the lexicographic method according to the average running time in seconds.

Additionally, the proposed method has been compared with the lexicographic method in terms of accuracy. The accuracy of the algorithm is computed by comparing the hospitals generated from each method to admit each patient with the correct hospital for admission identified manually. The accuracy can be mathematically represented as:

$$
\text { Acc }=\frac{\text { Total number of correctly directed patients }}{\text { Total number of patients' requests }} .
$$

Figure 4 presents the different comparisons between the two methods concerning the accuracy criterion. The evaluations have been made over different number of patients $(100,150,200,254)$ and different number of hospitals $(20,30,40,50,60)$. The figures show the high accuracy rates of the proposed method. The accuracy rates of the proposed method are very similar despite the number of patients and the number of hospitals. On-contrary, the lexicographic method accuracy rates suffer from a near steady decrease in the accuracy. This may return to its biasness to optimize one objective and provide a less degree of optimization for the other objectives.

From the previous discussion, it is clear that the admission problem is very critical to minimize the in-bed time of patients and to save the lives of patients with severe cases. The proposed method has identified two objective functions to formulate the admission problem. The method applied the $P O$ algorithm to vary among different hospitals according to the two objective functions. The method has been tested over a real-life dataset and proved its efficiency in admitting patients to suitable hospitals in real time. This presents the proposed method to be valid for real-life healthcare admission systems, especially for the second wave of Covid-19. 


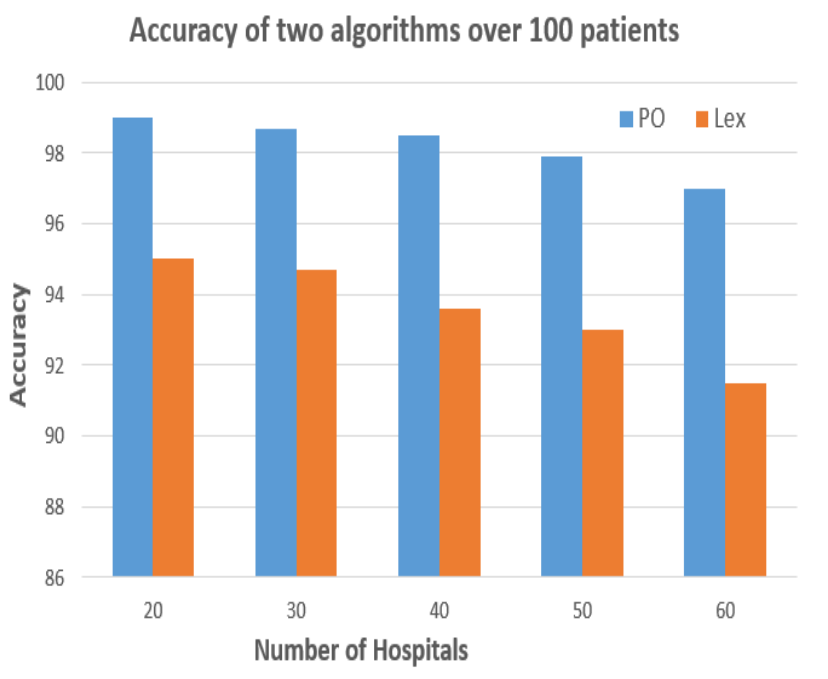

Accuracy of two algorithms over 200 patients

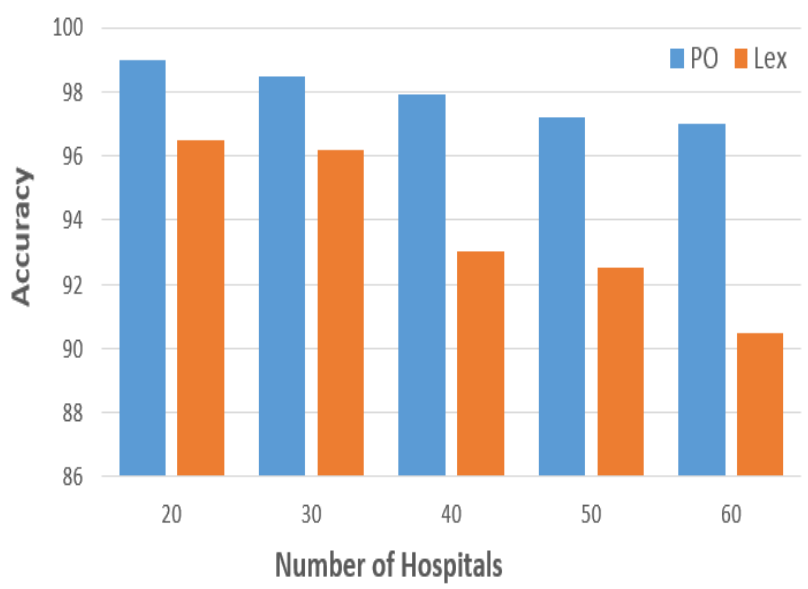

Accuracy of two algorithms over 150 patients

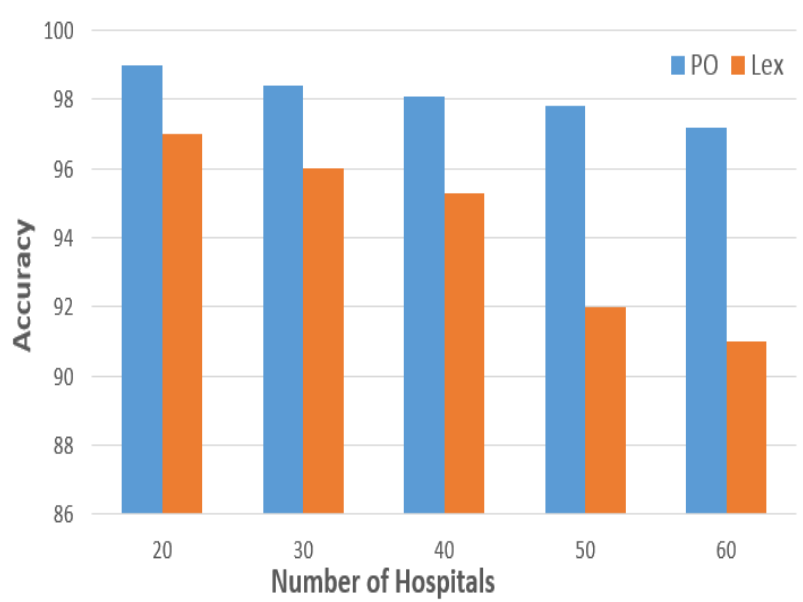

Accuracy of two algorithms over 254 patients

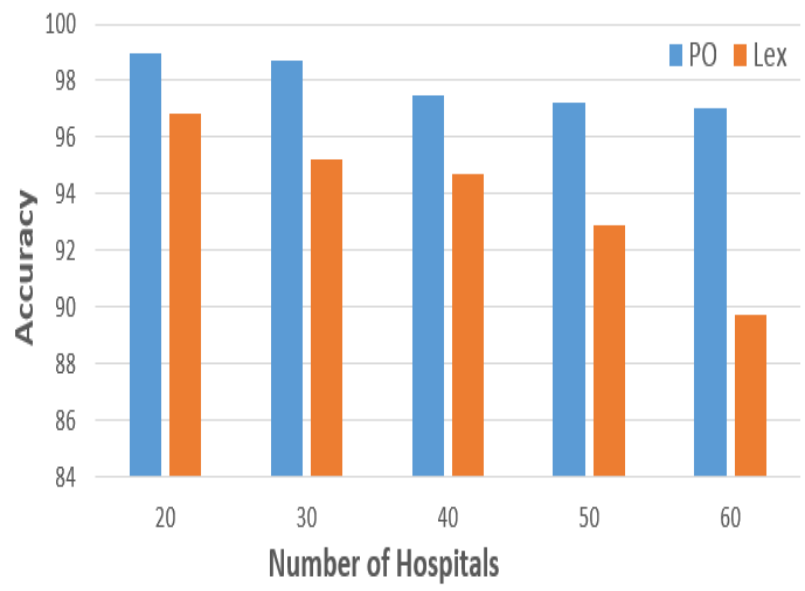

Figure 4. Comparisons between Pareto optimization and lexicographic algorithms according to accuracy over a different number of patients and hospitals.

\section{Conclusions}

Admitting patients to appropriate hospitals is an important problem. Optimizing this problem can save patients' lives and minimizes the in-bed time of patients in hospitals. Identifying the appropriate hospital for a patient concerning his medical status is one of the important objectives for the admission problem. Also, minimizing the waiting time of patients can save their lives. These two objectives have converted the admission problem into a Multi-Objective Problem (MOP). Pareto Optimization is a common method that has been used to solve MOPs. The proposed method employed PO to vary among different hospitals to choose the most suitable hospital based on the patient medical status. The method aimed to minimize the waiting time and to identify accurately the most suitable hospital for each patient. It was tested over a real-life dataset obtained from King Faisal hospital. The performance was evaluated according to admission time and accuracy. The proposed method was compared with the lexicographic multi-objective optimization method and presented its superiority concerning the two criteria. The authors aim to apply meta-heuristic methods to prepare the method to be applied to towns with a huge number of hospitals. 
Author Contributions: Conceptualization, L.A., S.B. and A.H.; Formal analysis, A.M.A. and L.A.; Funding acquisition, L.A.; Investigation, L.A.; Methodology, A.M.A. and A.H.; Project administration, A.H. and L.A.; Resources, L.A.; Supervision, L.A. and A.H.; Validation, L.A., S.B. and A.H.; Writingoriginal draft, A.M.A.; Writing-review \& editing, L.A., S.B. and A.H. All authors have read and agreed to the published version of the manuscript.

Funding: This research was funded by King Abdulaziz City for Science \& Technology (KACST) grant number 5-20-01-007-0006.

Acknowledgments: We would like to thank King Abdulaziz City for Science \& Technology (KACST), Kingdom of Saudi Arabia in collaboration with the Ministry of Health, the Saudi Health Council, and the Saudi Center for Disease Prevention and Control (SCDC) for generous Grant no. 5-20-01-007-0006. We want to declare that contributions and contents are original research that has not been published previously. We would like to thank in advance the anonymous reviewers for their valuable comments which helped us improve the content, quality, and presentation of this paper. We would like to extend our thank to Eisa Alanazi for sharing data with us.

Conflicts of Interest: All the authors declare no conflict of interest.

\section{References}

1. Bi, Q.; Wu, Y.; Mei, S.; Ye, C.; Zou, X.; Zhang, Z.; Liu, X.; Wei, L.; Truelove, S.A.; Zhang, T.; et al. Epidemiology and transmission of COVID-19 in 391 cases and 1286 of their close contacts in Shenzhen, China: A retrospective cohort study. Lancet Infect. Dis. 2020. [CrossRef]

2. Wu, Y.C.; Chen, C.S.; Chan, Y.J. The outbreak of COVID-19: An overview. J. Chin. Med. Assoc. 2020, 83, 217-220. [CrossRef]

3. Amin-Chowdhury, Z.; Aiano, F.; Mensah, A.; Sheppard, C.; Litt, D.; Fry, N.K.; Andrews, N.; Ramsay, M.E.; Ladhani, S.N. Impact of the COVID-19 Pandemic on Invasive Pneumococcal Disease and Risk of Pneumococcal Coinfection with SARS-CoV-2: Prospective national cohort study, England. Clin. Infect. Dis. 2020, 27, 1278-1286. [CrossRef]

4. Fujioka, S.; Fukae, J.; Ogura, H.; Mishima, T.; Yanamoto, S.; Higuchi, M.A.; Umemoto, G.; Tsuboi, Y. Hospital-based study on emergency admission of patients with Parkinson's disease. eNeurologicalSci 2016, 95, 51-67. [CrossRef]

5. Narzisi, G. Multi-Objective Optimization: A Quick Introduction; Courant Institute of Mathematical Sciences: New York, NY, USA, 2008.

6. Freitas, A.A. A Critical Review of Multi-Objective Optimization in Data Mining: A Position Paper. ACM SIGKDD Explor. 2004, 6,77-86. [CrossRef]

7. Mukhopadhyay, A.; Maulik, U.; Bandyopadhyay, S. A Survey of Multiobjective Evolutionary Clustering. ACM Comput. Surv. (CSUR) 2015, 47, 61. [CrossRef]

8. Parraga-Alava, J.; Dorn, M.; Inostroza-Ponta, M. A multi-objective gene clustering algorithm guided by apriori biological knowledge with intensification and diversification strategies. BioData Min. 2018, 18, 269-283. [CrossRef] [PubMed]

9. Ghany, K.K.A.; AbdelAziz, A.M.; Soliman, T.H.A.; Sewisy, A.A.E.M. A hybrid modified step Whale Optimization Algorithm with Tabu Search for data clustering. J. King Saud Univ. Comput. Inf. Sci. 2020. [CrossRef]

10. Kozan, R.B.E. A multi-criteria approach for hospital capacity analysis. Eur. J. Oper. Res. 2016, 255, 505-521. [CrossRef]

11. Liao, S.; Tu, H.; Hu, C.; Pan, W.; Xiong, J.; Yu, D.; Jing, L.; Pan, W. Fuzzy multi-objective medical service organization selection model considering limited resources and stochastic demand in emergency management. PLoS ONE 2019, 14, e0212308. [CrossRef]

12. Apornak, A.; Raissi, S.; Keramati, A.; Khalili-Damghani, K. Optimizing human resource cost of an emergency hospital using multi-objective Bat algorithm. Int. J. Healthc. Manag. 2020. [CrossRef]

13. Guedes, R.; Furtado, V.; Pequeno, T.; Rodrigues, J.J. Pareto set as a model for dispatching resources in emergency Centres. Peer-to-Peer Netw. Appl. 2019, 12, 865-880. [CrossRef]

14. Abedini, A.; Li, W.; Ye, H. An Optimization Model for Operating Room Scheduling to Reduce Blocking Across the Perioperative Process. Procedia Manuf. 2017, 10, 60-70. [CrossRef]

15. Benamrane, A.; Benelallam, I.; Bouyakhf, E.H. Constraint programming based techniques for medical resources optimization: Medical internships planning. J. Ambient. Intell. Humaniz. Comput. 2019. [CrossRef]

16. Zhang, J.C.L. Case Mix Index weighted multi-objective optimization of inpatient bed allocation in general hospital. J. Comb. Optim. 2017, 37. [CrossRef]

17. Kortbeek, N.; Braaksma, A.; Smeenk, F.H.; Bakker, P.J.; Boucherie, R.J. Integral resource capacity planning for inpatient care services based on bed census predictions by hour. J. Oper. Res. Soc. 2015, 66, 1061-1076. [CrossRef]

18. Nuñez-Perez, N.; Ortíz-Barrios, M.; McClean, S.; Salas-Navarro, K.; Jimenez-Delgado, G.; Castillo-Zea, A. Discrete-Event Simulation to Reduce Waiting Time in Accident and Emergency Departments: A Case Study in a District General Clinic. In Proceedings of the International Conference on Ubiquitous Computing and Ambient Intelligence, Philadelphia, PA, USA, 7-10 November 2017. [CrossRef] 
19. Gorunescu, S.B.F. Improving hospital bed occupancy and resource utilization through queuing modeling and evolutionary computation. J. Biomed. Inform. 2015, 53, 261-269. [CrossRef]

20. Anselmi, L.; Meacock, R.; Kristensen, S.R.; Doran, T.; Sutton, M. Arrival by ambulance explains variation in mortality by time of admission: Retrospective study of admissions to hospital following emergency department attendance in England. BMJ Open 2017. [CrossRef]

21. Conway, S.P.; Littlewood, J.M. Admission to hospital with asthma. Eur. PMC 1985. [CrossRef]

22. Pope, I.; Burn, H.; Ismail, S.A.; Harris, T.; McCoy, D. A qualitative study exploring the factors influencing admission to hospital from the emergency department. BMJ Open 2017. [CrossRef]

23. Kamila, N.K.; Jena, L.; Bhuyan, H.K. Pareto-based multi-objective optimization for classification in data mining. Clust. Comput. 2016, 19, 1723-1745. [CrossRef]

24. De Nardo, P.; Gentilotti, E.; Mazzaferri, F.; Cremonini, E.; Hansen, P.; Goossens, H.; Tacconelli, E. Multi-Criteria Decision Analysis to prioritise hospital admission of patients affected by COVID-19 in settings with hospital-bed shortage. Int. J. Infect. Dis. 2020, 494-500. [CrossRef] [PubMed]

25. Batista, A.; Vera, J.; Pozo, D. Multi-objective admission planning problem: A two-stage stochastic approach. Health Care Manag. Sci. 2020, 23, 51-65. [CrossRef] [PubMed]

26. Lott, A.; Haglin, J.; Belayneh, R.; Konda, S.R.; Egol, K.A. Admitting Service Affects Cost and Length of Stay of Hip Fracture Patients. Geriatr. Orthop. Surg. Rehabil. 2018, 9. [CrossRef]

27. Shuja, J.; Alanazi, E.; Alasmary, W.; Alashaikh, A. Covid-19 open source data sets: A comprehensive survey. Appl. Intell. 2020. [CrossRef]

28. Dunnett, S.; Leigh, J.; Jackson, L. Optimising police dispatch for incident response in real time. J. Oper. Res. Soc. 2018, 1476-9360. [CrossRef] 